\title{
METHODS OF CHANNELING SIMULATION
}

John H. Barrett

\author{
SOILID STATE DIVISION \\ OAK RIDGE NATIONAL LABORATORY \\ Operated by \\ MARTIN MARIETTA ENERGY SYSTEMS, INC. \\ Under \\ Contract No. DE-AC05-84OR21400 \\ With the \\ U. S. DEPARTMENT OF ENERGY \\ OAK RIDGE, TENNESSEE
}

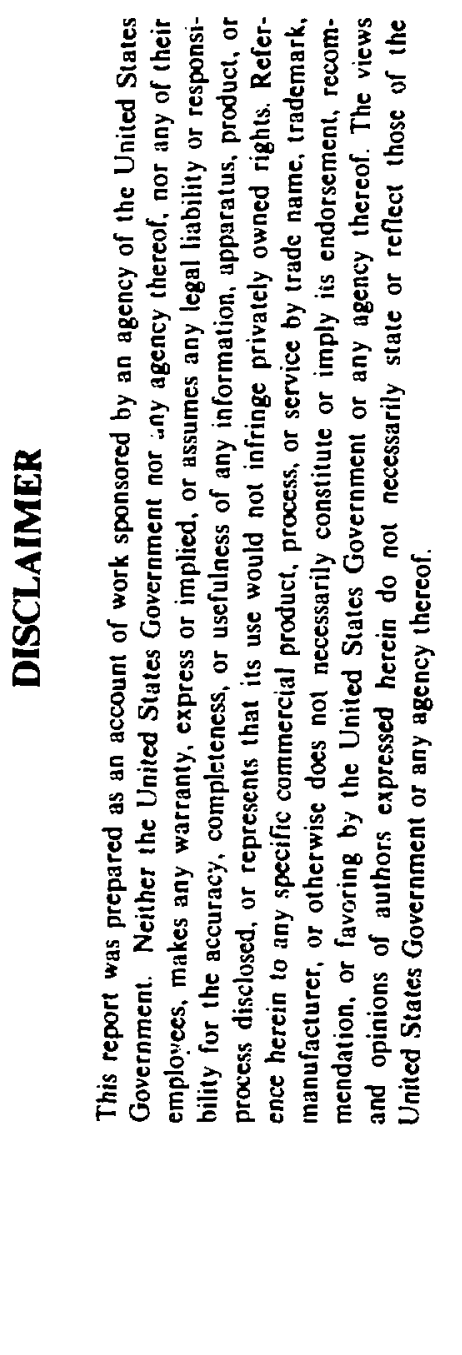

Junc 1989 


\title{
METHODS OF CHIANNELING SIMULATION
}

\author{
John H. Barrett \\ Solid State Division, Oak Ridge National Laboratory \\ Oak Ridge, Tennessee 37831-6032
}

\begin{abstract}
Many computer simulation programs have been used to interpret experiments almost since the first channeling measurements were made. Certain aspects of these programs are important in how accurately they simulate ions in crystals; among these are the manner in which the structure of the crystal is incorporated, how any quantity of interest is computed, what ion-atom potential is used, how deflections are computed froin the potential, incorporation of thermal vibrations of the lattice atoms, correlations of thermal vibrations, and form of stopping power. Other aspects of the programs are included to improve the speed; among these are table lookup, importance sampling, and the multiparameter method. It is desirable for programs to facilitate incorporation of special features of interest in special situations; examples are relaxations and enhanced vibrations of surface atoms, easy substitution of an alternate potential for comparison, change of row directions from layer to layer in strainedlayer lattices, and different vibration amplitudes for substitutional solute or impurity atoms. Ways of implementing all of these aspects and features and the consequences of them will be discussed.
\end{abstract}


Channeling was dirst observed in calculations of atoms slowing down in crystalline solids by Robinson and Oen [1]. Thereby, theirs became the first channeling simulation program; since that time, many others have been written [2-9]. This paper will offer a brief review and explanation of the methodology involved in the simulation of channeling of $\mathrm{H}$ and heavier ions having energies of $50 \mathrm{keV} / \mathrm{amu}$ or above; no attempt will be made to cover the related fields of channeling calculations using semianalytic methods or continuum potentials or of the simulation of lower energy ion scattering phenomena or collision cascades. The following sections will discuss basic features, efficiency features, applications features, and possible future directions of such programs. Examples will mostly be pointed out by reference to published work.

Channeling involves the interplay of nuclear and atomic physics and of many subfields of solid state physics; this multifaceted nature makes it a stimulating field to work in. It also means that simulation of channeling must take into account these numerous components of the physics involved.

\section{Basic features}

It has been shown that the scattering of $\mathrm{H}$ and heavier particles with energies in the keV range and abov'e can be treated classically [10]. Within the classical approach several aspects of the problem are fundamental enough that they should be included in som.e manner in a channeling simulation program. Those aspects that have been discussed by the author previously [3] will be presented only briefly here; others will be presented at greater length.

The most basic feature is the incorporation of the lattice structure. Since channeling by rows is such a striking aspect of the phenomenon, most programs $[2-5,7-9]$ have viewed the lattice as composed of rows. This view 
will be sound as long as the ion beam is directed reasonably close to a rnajor row direction. In this approach the program can take advantage of the fact that the next important collision will generally be with the next atom along a row from the last one. It is only necessary to check for those occasions when a trajectory moves from the vicinity of one row to that of another and to transfer attention to the new row before continuing. During the transfer of attention, proper track must be kept of the atomic positions in the new row relative to those of the previous one. The author's program LAROSE, based on this lattice-of-rows concept, is capable of doing simulations for three major row directions in all elements having face-centered cubic, body-centered cubic, or diamond lattices. A row-oriented procedure affords a good combination of accuracy and efficiency. It has the disadvantage that each row direction in each lattice requires separate programming effort.

An alternate procedure $[1,6]$ to incorporate the lattice structure is to provide the program with the basic information or: the location of atoms in the unit cell and the translational properties of the crystal and let the program perform a search procedure to find the next lattice atom or atoms with which the projectile will interact. A search-oriented procedure has the advantage of affording more ease in selecting crystals and beam directions for simulation. It has the disadvantage of being less efficient, and it may not allow distant collisions to be taken into account as thoroughly.

Some other features that the program must provide are the proper distribution of starting positions, the divergence of the beam around its central direction, and mosaic spread of the crystal when these latter two factors are important. These have been implemented sometimes by using a random number generator and at other times by using a grid of uniformly 
spaced points. Neither has been shown to be more accurate than the other, and the choice is based on preference.

The ion-atom interaction must be based on a sufficiently accurate potential energy function for the screened Coulomb interaction. No single form of the screening function has been shown to be definitely superior to all others. Molière's approximation [11] to the Thomas-Fermi screening function is the most widely used since it provides a universal function and is reasonably accurate. Lindhard's standard potential [10] has also been used frequently because its form allows relatively rapid calculation directly from a formula. The calculation of the deflection of an ion which has a given impact parameter on a lattice atom is usually done by the impulse approximation [12], which gives good accuracy [3]. Numerical integration will also be highly accurate if properly done.

If deflections are calculated for only the atoms in the nearest row in the row-oriented procedure described above, the simulation is a binary collision one. This can be implemented very easily by cutting off the potential or the deflection at a radius equal to or less than the size of the region associated with one row. For many purposes the binary collision approximation will be adequate; often, however, it will be desirable to go beyond this approximation. A scheme used in LAROSE for including the deflections due to other rows is illustrated in Fig. 1. The rhombus surrounding the central row in the figure is the cross-sectional area assigned to that row; the set of rhombuses for all of the rows fills the whole plane. The crossing of the boundary of the rhombus signals the time to transfer attention to another row as the central une. For the central row the thermal vibration of each atom is treated in the manner to be described in the next paragraph; for the surrounding rows the vibrations are taken into account by averaging their continuum potential energy over 
the vibration distribution [13]. Computation of the deflection due to a row of atoms from its continuum potential is intimately connected with the impulse approximation [12]. Inasmuch as a table lookup procedure has advantages in computing speed, as will be discussed in the next section, a table is computed incorporating the deflections of all rows that are important. In order to handle the many "other rows" conveniently, they are thought of as being in rings surrounding the central row; the first two are shown in Fig. 1. The numbers of rows contained in successive rings are $8,16,24,32$, etc. The number of other-row rings to be included in a calculation was selected by requiring that the deflection due to any neglected row be less than the deflection for the most important row by at least five ordars of magnitude; it varies with each combination of target and row direcion. The appropriate numbers of other-row rings are stored in LAROSE for the various combinations. This other-row feature was not included in the version described in Ref. 3 but was incorporated in a new version soon afterwards. Its absence would not affect any of the qualitative results in Ref. 3; the one quantitative result that might be affected would he the formula for planar half angles since planar channeling would be more affected than would be axial channeling. The later version incorporating the other-row feature was used to prepare later papers by the author, in particular those dealing with planar channeling $[14,15]$.

Realistic thermal vibrations must be included if simulation results are to be adequate [16]. The procedure usually used is to give each lattice atom an independent random displacement by using a random number generator to choose each of the three components of the displacement at random from a Gaussian distribution having a width computed from the Debye theory. The Debye temperature used should be that appropriate to the Debye-Waller factor 
rather than to the specific heat, although the latter can be used when the former is unknown. There is no general compilation of Debye-Waller temperatures, but values for a particular crystal can generally be found in the literature. Correlations in the vibrations of adjacent lattice atoms can be introduced by a procedure due to Jackson [17]; their effect is equivalent to a reduction of the vibration amplitude of about 5 to $10 \%$ depending on the effect being looked at. The small size of the correlation effect is fortunate in view of the general unavailability of the correlation parameters. However, it would be desirable to see correlation effects incorporated in channeling simulations more generally than they are.

For many applications it is not necessary to take account of the energy loss that ions undergo during their passage througn a crystal as long as the fraction of energy lost is not large; for some purpcses, however, inclusion of this loss is important. Whenever the energy loss is of significance, a theory should be used that includes the dependence on impact parameter since different channeled or near-channeled trajectories sample different values of impact parameter. The form that has been most frequently used is one suggested by Oen and Robinson [18]. Further theoretical development may be needed to arrive at a completely satisfactory form for the energy loss.

A wide variety of output quantities can be computed from the simulated ion trajectories. The most important has proved to be the nuclear encounter probability defined by Eq. (5) of Ref. 3; this gives the probability of close encounter events such as a nuclear reaction, a large angle scattering of an ion, or a high-energy recoil of a lattice atom. The use of this quantity makes channeling calculations much more efficient than blocking calculations so that blocking is always replaced by channeling whenever possible by 
using the principle of reciprocity [10]. By dividing a group of trajectories up into batches, an estimate of accuracy can be given for the result.

In calculating the nuclear encounter probability, the simplest method is to evaluate the contribution at each collision or for each trajectory in a synchronous way as they occur. However, a most ingenious alternative has been developed [6] in which the trajectories are all stored and the calculation deferred until later. This gives one approach to performing double alignment calculations and also makes possible the multiparameter method to be described in the next section. The program described in Ref. 6 couples the choices of a search-oriented procedure and the deferred method of calculation, but either could be selected independently of the other.

There are other forms of output that can be important at times. In general, direct plotting of trajectories provides an indigestible amount of detail, but at times it can be used to reveal aspects of channeling [19] that would be difficult to demonstrate in any other way. A form of output that is useful in interpreting atom location measurements $[8,20-1]$ is a histogram of ion flux across the channel; for this purpose the rhombus in Fig. 1 can be mapped onto a rectangle. One other form of output often of interest is the pattern of emergent ion directions as they leave the crystal.

There is one general feature that is common to all of the foregoing. Whenever a choice needs to be made about how much effort to put into program development or whether extra computing time is justified, it is best to accept any reasonable amount of effort or time. This approach not only gives confidence in the program but often avoids attention to the same matter at a later time.

The final part of developing a simulation program is testing. One feature that the author has found of continuing use is an option that has 
L' $\wedge^{\wedge}$ ROSE print collision-by-collision details of trajectories. This feature must, of course, be used with a very limited number of trajectories, but it affords a chance to check that the program is performing as intended. It can continue to be of use whenever modifications are made. Other checks can be performed, such as verifying that one obtains unity for the nuclear encounter probability in a "random" direction with large thermal vibrations and unity for the ion flux distribution averaged over the whole channel.

One check of the calculations is worthy of particular mention; it concerns the directions of trajectories that are planar channeled. Various aspects of calculating the deflections of atoms in the central row and the other rows can cause a bias towards deflections that are very slightly towards or away from the nearest major row direction. In all versiors of LAROSE the * author has always found and sought to remove or at least minimize such biases. A very good way to look for them is to do calculations for planar channeling in a major set of planes at an appreciable angle to the nearest major row direction and look for shifts of the beam spot away from the expected direction; Fig. 2 gives two examples. Part (a) shows the results for the current version of LAROSE including the deflections due to other rows as well as the central row, while part (b) shows a similar calculation but with the deflections due to other rows omitted. Although the pattern in (a) does have a very slight spot shift, it is typical of planar channeling; there is an intense elliptical central spot with a more diffuse hour-glass shaped pattern extending above and below it. The pattern in (b) shows a striking spot shift involving distortion into a crescent shape. In addition to the shift and distortion, the very different distribution of intensity along the direction transverse to the plane means that the planar channeling oscillations of the trajectories are different because the potential has been changed by having the contributions 
from the other rows removed. The incorrect calculation of the planar channeling oscillations in Fig. 2(b) is a direct result of the cutoff in the deflection of the ions by the lattice atoms at a distance at which it is still appreciable. It is probable that the amount of spot shift could be reduced considerably by changing slightly the criterion for when the shift is made from one row to another as being the central one; however, that would not change the deficiency in the planar potential because the contributions from the other rows would still be missing. The magnitude of the spot shift increases with increasing depth into the crystal and with increasing angle of the beam from the major row direction. In the author's experience this problem of spot shift has been so all pervasive that it should always be tested for in a channeling simulation program down to the maximum depth for which it is to be used. In addition, tests should be made of whether any cutoff distance for deflection of ions by the lattice atoms will affect any significant aspect of the calculation. The author has only had experience with these questions for row-oriented procedures for incorporating the lattice structure, but it is very likely that the same effects can exist for search-oriented procedures.

\section{Efficiency features}

There are some features of a program that are not necessary to its accuracy but which may be desirable to increase its efficiency; among these are table lookup. One table used in LAROSE is made up of 1000 entries that have a Gaussian distribution. It is used in connection with a random number generator to select thermal vibrations or other quantities that are to be chosen at random from a Gaussian distribution. By replacing an alternate procedure that was previously used, about $15 \%$ in computing time is saved. 
Another place in which a table lookup can improve efficiency is in calculating the deflection of an ion at a collision as a function of its impact parameter. By replacing the previously used sum over three modified Bessel functions by a quadratic table lookup procedure, approximately another $15 \%$ in computing time is saved. By constructing the table for the reciprocal of the deflection angle, accuracy to near machine precision can be obtained with a table of reasonable size while using the same interval over the whole range. Since a table was already in use for the deflections due to atoms in the central row, it was very natural to adopt a table lookup procedure (also quadratic) for the deflections due to other rows. Although the small amount of time to set up the other-row table is proportional to the number of rows, the later use of the table is independent of that number. Once a table lookup procedure is in use, changing the potential used is just a matter of changing the formula used to create the table. Table lookup is a much more accurate way of obtaining ffificiency than an approximate formula.

When a simulation is done for the ion beam aligned with a channel, contributions to the nuclear encounter probability come only from those trajectories that start sufficiently close to the rows or planes that bound the channel. This is an example of a general situation in which importance sainpling can increase the efficiency of a calculation. Ways of doing importance sampling that the author has developed are illustrated in Fig. 3 for axiai and planar channeling. As shown in part (a), the way of doing this for a row is to use a series of annular rings surrounding the row as starting points for different groups of trajectories; it is ge'ierally convenient to scale the size of the rings to the thermal vibration amplitude. Before doing a calculation, the author uses trial-and-error to determine how many rings to use, ring sizes, and how many trajectories to assign to each for optimum 
results. For planar channeling, bands of starting points as shown in part (b) are adopted; the bands are generally paired as shown, with the starting points of trajec-tories alternating between them. This importance sampling method will generally save a factor of over 10 in computing time in the axial case and of 2 to 4 in the planar case.

A main use to which the deferred method of calculation [6] has been put is to utilize a single batch of stored trajectories with many sets of parameters that must be explored when ion channeling is used to study the structure of solid sitrfaces; this has been named the multiparameter method. Although the deferred method is slower than the synchronous method for any one set of parameters, it becomes considerably faster when used with a very large number of sets of parameters.

\section{Applications features}

A desirable property of a channeling simulation program is that it he easy to introduce special features in order to do calculations of particular interest; indeed, most applications probably do involve some special feature. Fortunately, the division of a program into a logical format of subroutines provides a good starting point for the needed changes. Certain special features were used in the basic work [3] to explore critical angles, minimum yields, and dependences on energy, temperature, and depth. Numerous other variations of LAROSE have been used; some, such as the importancesampling procedure described in the preceding section and the flux histogram for atom location, proved to be of such general usefulness that they were incorporated permanently into it. Probably the most widely used variant has been one for doing channeling calculations related to surface structure, which may involve surfaces with overlayers, relaxations, and enhanced vibrations 
[20,22]; surface calculations have been a very fertile field for others [23-4] as well. Other variants of LAROSE have been used for measurement of nuclear lifetime by blocking [25], study of inter-row focusing which causes a breakdown of the statistical equilibrium hypothesis in channeling [19], method for choosing between potentials and studying the stopping power of channeled ions $[13,14]$, evaluation of correlations of thermal vibrations [17], help in understanding channeling radiation [26], study of enhanced backscattering near $180^{\circ}$ [27], applications of channeling in strained-layer lattices [28-9], and measurement of difference in vibration amplitudes between solute and host atoms in an alloy [30]. This list of applications is not meant to be complete, but rather to give a good indication of the variety of channeiing situations to which simulation has been applied.

\section{Future directions}

There are at present some gaps in our knowledge of items that affect simulations of channeling. One concerns correlations of thermal vibrations. A large body of data on neutron inelastic scattering by lattice vibrations and the theory to calculate correlations from that data exist, but very little use has been made of it. Consequently, correlation parameters exist in only a very few cases and even ther are generally unpublished. There is considerably more information on Debye-Waller temperatures, but there is no tabulation or critical evaluation of competing results. Theory on energy loss as a function of impact parameter exists, but work needs to done to assess its adequacy for channeling calculations and put it in a convenient form for use. Finally, it would be desirable to establish what is the best potential energy function to use for each element or establish that the exact form of potential to use is not critical and that some group of poientials are all satisfactory. 
Much current interest in channeling is in materials that have more complicated structures, such as high temperature superconductors, than those that have been simulated in the past. In order for a row-oriented procedure for complicated structures to be developed, it will probably be necessary to create computer programs that perform the analysis of a lattice structure in terms of rows and the relationships of the rows to one another. An auxilliary program might be an aid in creating the main program or might be made part of the setup procedure in the program; current computer graphics and interactive systems should facilitate such a development. If a search-oriented procedure is to be used, the size of its search region would need to be large, and checking would need to be done to insure that any cutoff distance would not affect accuracy.

As the trend towards ever greater computing speed and storage at ever lower cost continues, current microcomputers have reached the stage at which they will run a channeling simulation program quite well if they have an adequate compiler. One can anticipate that simulations will come increasingly to be run on such machines in the future. The availability of greater computing power may facilitate programs having new features, such as expanded sizes of search regions in search-oriented procedures. Vector processing is not a computing feature that is useful for channeling simulations since these programs do not vectorize. Parallel processing, however, is a feature that is ideally suited to channeling simulation with each processor running a separate batch of trajectories; very little intercommunication would be needed between the processors. One requirement would be a random number generator that would supply independent sequences of random numbers to each processor. 


\section{ACKNOWLEDGMENT}

This research was sponsored by the Oak Ridge National Laboratory under USDOE contract DE-AC05-84OR21400 with Martin Marietta Energy Systems, Inc. 


\section{References}

[1] M. T. Robinson and O. S. Oen, Phys. Rev. 132 (1963) 2385.

[2] J. H. Barrett, Phys. Rev. 166 (1968) 219.

[3] J. H. Barrett, Phys. Rev. B 3 (1971) 1527.

[4] D. '. Morgan and D. van Vliet, Can. Jour. Phys. 46 (1と68) 503.

[5] I. Stensgaard, L. C. Feldman, and P. J. Silverman, Surf. Sci. 77 (1978) 513.

[6] R. M. Tromp and J. F. van der Veen, Surf. Sci. 133 (1983) 133; J. W. M. Frenken, R. M. Tromp, and J. F. van der Veen, Nucl. Instr. and Meth. B17 (1986) 334.

[7] B. Schmiedeskamp, P. Jonk, H. E. Roosendaal, and H. O. Lutz, Nucl. Instr. and Meth. B17 (1986) 309.

[8] P. J. M. Smulders and D. O. Boerma, Nucl. Instr. and Meth. B29 (1987) 471.

[9] A. Dygo and A. Turos, Phys. Lett. A 127 (1983) 281.

[10] J. Lindhard, Kgl. Danske Videnskab. Selskab, Mat.-Fys. Medd. 34, (1965) No. 14.

[11] G. Molière, Z. Naturforsch. 2a (1947) 133.

[12] C. Lehman and G. Leibfried, Z. Physik 172 (1963) 465; C. Lehman and G. Leibfried, J. Appl. Phys. 34 (1963) 2821.

[13] See the Appendix of Ref. 3.

[14] J. H. Barrett, Phys. Rev. B 20 (1979) 3535.

[15] R. J. Culbertson, S. P. Withrow, and J. H. Barrett, Nucl. Instr. and Meth. B2 (1984) 19.

[16] T. S. Noggle and J. H. Barrett, phys. stat. sol. 36 (1969) 761.

[17] J. H. Barrett and D. P. Jackson, Nucl. Instr. and Meth. 170 (1980) 115.

[18] O. S. Oen and M. T. Robinson, Nucl. Instr. arid Meth. 132 (1976) 647. 
[19] J. H. Barrett, Phys. Rev. Lett. 31 (1973) 1542.

[20] J. H. Barrett, Nucl. Instr. and Meth. 149 (1978) 341.

[21] A. D. Marwick, G. S. Oehrlein, J. H. Barrett, and N. M. Johnson, p. 259 in Defects In Electronic Materials, ed. by Michael Stavola, S. J. Pearton, and G. Davis, Materials Research Society, Pittsburgh, 1988.

[22] D. M. Zehner et al., J. Vac. Sci. Technol. 12 (1975) 454; L. C. Feldman et al., Phys. Rev. Lett. 39 (1977) 38; R. J. Culbertson et al., Surf. Sci. 157 (1985) 451; S. P. Withrow et al., Surf. Sci. 161 (1985) 584.

[23] J. F. van der Veen, Surf. Sci. Rep. 5 (1985) 199.

[24] Papers in Workshop at this Conference on IBA of SurfacesReconstruction and Relaxation.

[25] Y. Hashimoto, J. H. Barrett, and W. M. Gibson, Phys. Rev. Lett. 30 (1973) 995.

[26] M. J. Alguard et al., Nucl. Instr. and Meth. 172 (1980) 7.

[27] B. R. Appleton, O. W. Holland, and J. H. Barrett, Nucl. Instr. and Meth. 191 (1981) 507.

[28] J. H. Barreit, Phys. Rev. B 28 (1983) 2328.

[29] C. K. Pan et al., Phys. Rev. B 31 (1985) 1270.

[30] J. H. Barrett, Phys. Rev. B 38 (1@88) 5069. 


\section{Figure Captions}

Fig. 1. Arrangement of [110] rows in a face-centered cubic lattice with lattice constant $g$.

Fig. 2. Beam cross section for $1 \mathrm{MeV}$ He in Au (111) planes at a depth of $0.10 \mu \mathrm{m}$ : (a) with and (b) without other-row deflections. Angles are relative to the beam direction at the surface, which was $13.1^{\circ}$ from [110].

Fig. 3. Regions used to start trajectories having higher or lower probabilities of interaction: (a) for rows and (b) for planes. 


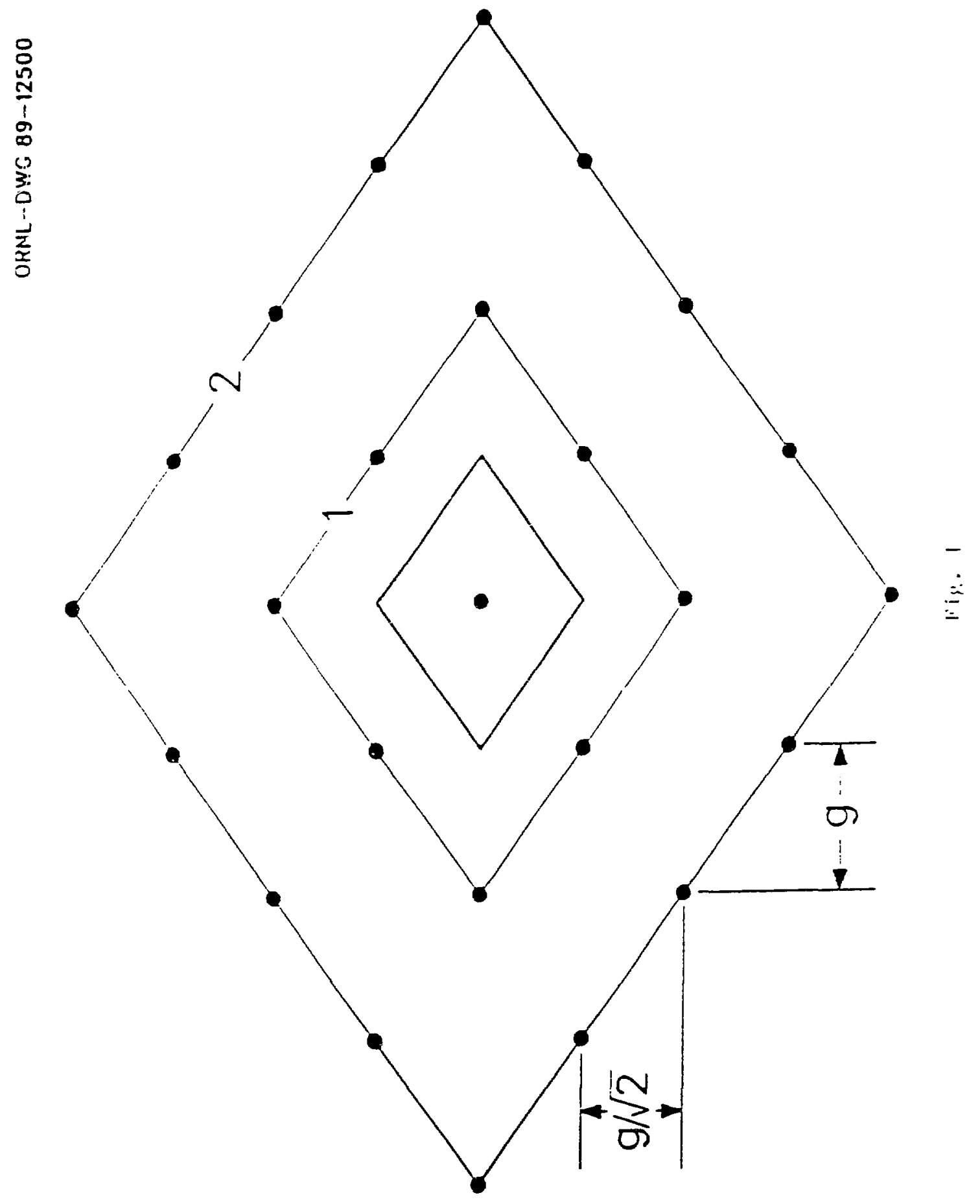


ORNL-DWG 89-12501

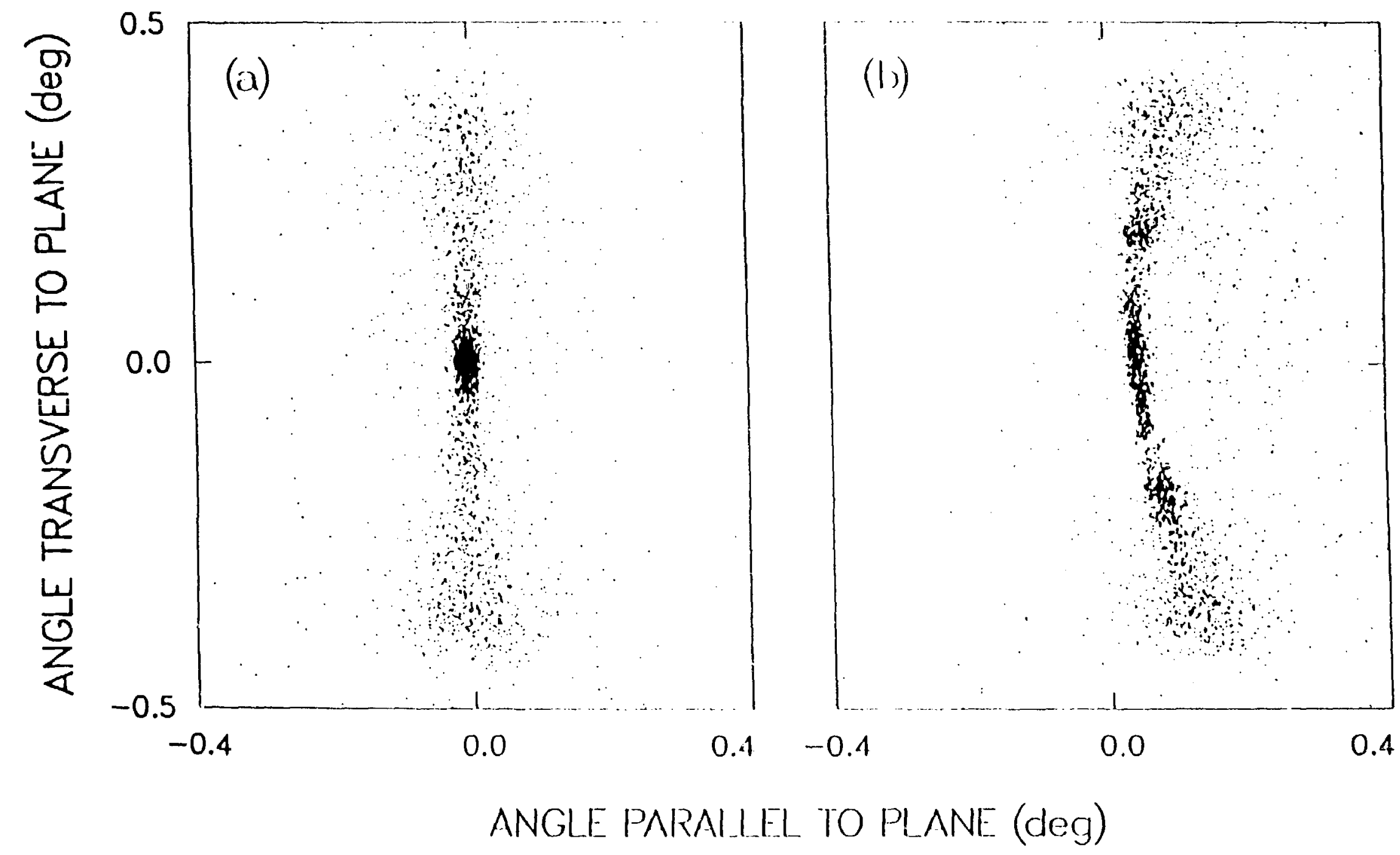

Fig. 2 


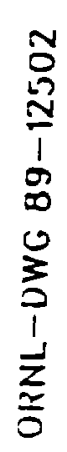
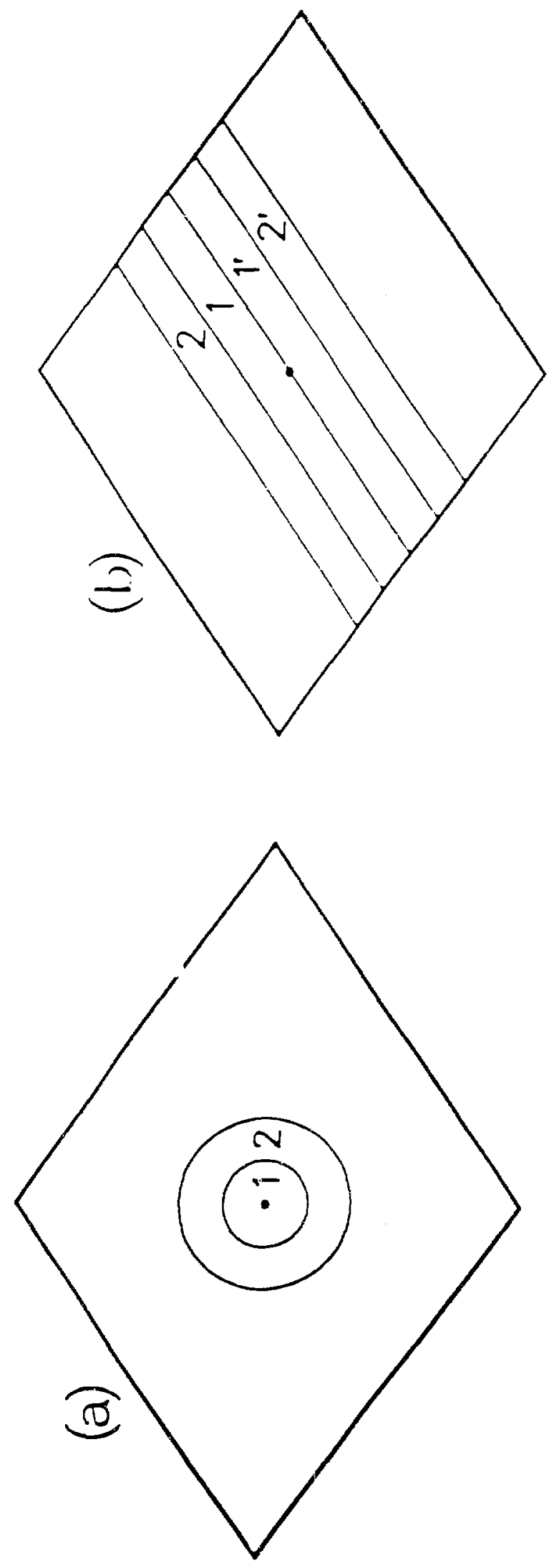